\title{
Evolution of substance use, neurological and psychiatric symptoms in schizophrenia and substance use disorder patients: a 12-week, pilot, case-control trial with quetiapine
}

\section{Simon Zhornitsky ${ }^{1}$ Emmanuel Stip ${ }^{1}$, Joelle Desfossés ${ }^{1}$, Tania Pampoulova ${ }^{1,2}$, Élie Rizkallah ${ }^{1,2}$, Pierre-Paul Rompré ', Lahcen Aït Bentaleb ${ }^{1}$, Olivier Lipp ${ }^{1}$, Jean-Pierre Chiasson ${ }^{2}$, Alain Gendron ${ }^{3}$ and Stéphane Potvin ${ }^{1 *}$}

1 Fernand-Seguin Research Centre, Department of Psychiatry, Faculty of Medicine, Université de Montréal, Montréal, QC, Canada

2 Clinique du Nouveau-Départ, Montréal, OC, Canada

${ }^{3}$ AstraZeneca Pharmaceuticals, Mississauga, ON, Canada

\section{Edited by:}

Lorenzo Leggio, Brown University, USA

\section{Reviewed by:}

Giovanni Martinotti, Catholic University of Rome, Italy

David Gorelick, National Institute on

Drug Abuse, USA

${ }^{*}$ Correspondence:

Stéphane Potvin, Centre de recherche

Fernand-Seguin, 7331 Hochelaga,

Montréal, QC, Canada H1N 3V2.

e-mail: stephane.potvin@umontreal.ca
Neurological and psychiatric symptoms are consequences of substance abuse in schizophrenia and non-schizophrenia patients. The present case-control study examined changes in substance abuse/dependence, and neurological and psychiatric symptoms in substance abusers with [dual diagnosis (DD) group, $n=26$ ] and without schizophrenia [substance use disorder (SUD) group, $n=24$ ] and in non-abusing schizophrenia patients (SCZ group, $n=23$ ) undergoing 12-week treatment with the atypical antipsychotic, quetiapine. Neurological and psychiatric symptoms were evaluated with the Positive and Negative Syndrome Scale, the Calgary Depression Scale for Schizophrenia, the Extrapyramidal Symptoms Rating Scale, and the Barnes Akathisia Rating Scale. At endpoint, DD and SCZ patients were receiving significantly higher doses of quetiapine ( mean $=554$ and $478 \mathrm{mg} /$ day, respectively), relative to SUD patients (mean $=150 \mathrm{mg} /$ day). We found that SUD patients showed greater improvement in weekly dollars spent on alcohol and drugs and SUD severity, compared to DD patients. At endpoint, there was no significant difference in dollars spent, but DD patients still had a higher mean SUD severity. Interestingly, DD patients had significantly higher parkinsonism and depression than SCZ patients at baseline and endpoint. On the other hand, we found that SUD patients had significantly more akathisia at baseline, improved more than SCZ patients, and this was related to cannabis abuse/dependence. Finally, SUD patients improved more in Positive and Negative Syndrome Scale positive scores than DD and SCZ patients. Taken together, our results provide evidence for increased vulnerability to the adverse effects of alcohol and drugs in schizophrenia patients. They also suggest that substance abuse/withdrawal may mimic some symptoms of schizophrenia. Future studies will need to determine the role quetiapine played in these improvements.

Keywords: schizophrenia, substance use disorder, paranoia, akathisia, quetiapine, cannabis

\section{INTRODUCTION}

Schizophrenia is the most disabling psychiatric disorder, according to the Global Burden of Disease study (Eaton et al., 2008). Important contributors to disability in schizophrenia are psychiatric (e.g., positive, negative, and depressive symptoms) and neurological symptoms (e.g., parkinsonism, dyskinesia, and akathisia; Patterson et al., 1998; Villalta-Gil et al., 2006; Aubin et al., 2009). Compounding these problems is the nearly $50 \%$ lifetime prevalence of substance use disorder (SUD) associated with schizophrenia (Regier et al., 1990). In non-psychotic individuals, substance use is associated with neurological and psychiatric symptoms (Mauri et al., 2007; Zhornitsky et al., 2010a). In schizophrenia patients, substance use has a negative impact on the course of the pathology. Compared to non-abusing patients, dual diagnosis (DD) schizophrenia patients are more frequently hospitalized, non-compliant with treatment, suicidal, impulsive and violent, homeless and unemployed, and they have more legal and health problems (Mueser et al., 1998; Negrete, 2003). Similarly, there is evidence that DD patients have more neurological and psychiatric symptoms than non-abusing schizophrenia patients (Bersani et al., 2005; Potvin et al., 2007, 2009; Harrison et al., 2008).

Current evidence suggests that atypical antipsychotic treatment is associated with improvements in psychiatric symptoms in schizophrenia (Lieberman et al., 2005; Lee et al., 2009; Nakamura et al., 2009). Due to these benefits, as well as their low propensity to induce neurological symptoms, atypical antipsychotics are increasingly being tried as treatments for substance abuse in psychotic and non-psychotic patients (for review, see Zhornitsky et al., 2010b). Indeed, previous studies in singleand DD patients suggest that atypical antipsychotics may lead to improvements in alcohol use disorder (Littrell et al., 2001; Martinotti et al., 2007, 2009). Some studies have also found atypical antipsychotics to improve cannabis use disorder in DD patients (Green et al., 2003; van Nimwegen et al., 2008). However, irrespective of their efficacy for actually relieving substance abuse, we know very little about the effects of atypical 
antipsychotics on neurological and psychiatric symptoms when prescribed to substance abusers with or without comorbid psychosis. This is an important area of study because any residual symptoms and deficits may act as negative reinforcers to maintain the cycle of addiction (Koob and Le Moal, 2001), and may impair their social functioning and quality of life (Addington and Addington, 1997; Lahmek et al., 2009).

The present study examined substance use outcomes and neurological and psychiatric symptoms in substance abusers with and without schizophrenia and in non-abusing schizophrenia patients undergoing a 12-week treatment with the atypical antipsychotic quetiapine. This antipsychotic was chosen because it has previously been shown to improve substance use outcomes in psychotic and non-psychotic patients (Potvin et al., 2006a; Kampman et al., 2007; Martinotti et al., 2008; Rizkallah et al., 2010) and is an effective monotherapy for anxiety and depressive disorders (for review, see Zhornitsky et al., 2011), while also producing little or no neurological symptoms (Weiden, 2007). Importantly, this is the first study of its kind to trace the evolution of neurological and psychiatric symptoms in all three groups of patients undergoing a homogenous antipsychotic treatment. This study is complementary to earlier studies by Potvin et al. (2006a) and Rizkallah et al. (2010), which reported substance abuse and clinical outcomes for DD patients and non-schizophrenia substance abusers.

\section{MATERIALS AND METHODS \\ PARTICIPANTS}

Three groups of participants were recruited, namely: (i) substanceabusing patients with schizophrenia-spectrum disorders (schizophrenia, schizoaffective disorder, schizophreniform disorder; DD group); (ii) non-psychotic substance abusers in detoxification (SUD group); and (iii) schizophrenia patients with comorbid substance abuse (SCZ group). Psychiatric and SUD diagnoses were by well-trained psychiatrists (Lahcen Ait Bentaleb, Olivier Lipp, and Emmanuel Stip) and physicians (Jean-Pierre Chiasson), and were all based on DSM-IV criteria. SUD diagnoses were complemented with urine drug screenings. In the SUD group, there were two diagnoses of borderline personality disorder and two diagnoses of substance-induced psychotic disorder (DSM-IV). All participants signed a detailed consent form. The study was approved by the local ethics committee.

For all three groups, exclusion criteria were: (i) patients already on clozapine or quetiapine; (ii) patients hospitalized in a psychiatric unit; (iii) pregnancy; (iv) female subjects of childbearing potential or inadequate contraception; and (v) clinically meaningful unstable, renal, hepatic, cardiovascular, respiratory, cerebrovascular, or other serious, progressive physical disease. For the DD and SCZ groups, patients were excluded if their total score on the Positive and Negative Syndrome Scale (PANSS; Kay et al., 1987) was lower than 65. Adjuvant medications were allowed in all three groups.

\section{CLINICAL ASSESSMENTS}

Neurological symptoms were evaluated with the Extrapyramidal Symptoms Rating Scale (ESRS; Chouinard et al., 1980). Akathisia was evaluated with the Barnes Akathisia Scale (BAS; Barnes, 1989). Psychiatric symptoms were measured using the PANSS and the
Calgary Depression Scale for Schizophrenia (CDSS; Addington et al., 1993). For more information on clinical assessments, refer to Potvin et al. (2006a).

\section{SUD ASSESSMENTS}

Quantities of substances used in the last week were also registered, using the TimeLine Follow-Back (TLFB) procedure (Sobell and Sobell, 1992). Quantities used were noted for all substances. Amount spent on substances was calculated based on the value market in Quebec province (Canada). To complement our evaluation of SUDs, urine screenings were performed on weeks 0 and 12, for cannabinoids, opiates, and psychostimulants. SUD severity was also evaluated using an adapted eight-item scale, based on DSM-IV criteria of substance dependence. Two trained students and a trained nurse scored [from 0 (no problem) to 5 (severe problem)] the patient's SUD severity on the following items: (1) loss of control; (2) time spent on PAS; (3) impact of SUDs on social life; (4) impact of SUDs on daily occupations; (5) physical impact of SUDs; (6) psychiatric impact of SUDs; (7) impact of SUDs on compliance; and (8) ability to enjoy pleasures other than substance use. For more information on SUD assessments, refer to Potvin et al. (2006a) and Rizkallah et al. (2010).

\section{STATISTICAL ANALYSES}

Baseline and endpoint differences between the DD, SCZ, and SUD groups were analyzed using one-way analyses of variance (ANOVA) with group as the independent variable. Changes in substance abuse, neurological and psychiatric symptoms were analyzed using mixed ANOVA with group as the independent variable and time as the repeated measure. Multiple comparisons were performed using the Bonferroni correction. The influence of potential confounds on improvements in neurological and psychiatric symptoms were analyzed using analyses of covariance (ANCOVA). Dichotomous variables were evaluated using Pearson's Chi-square test. The level of significance was set at $p<0.05$. Last-observation carried forward (LOCF) was used. Statistical analyses were performed using the Predictive Analytics SoftWare (PASW; version 18).

\section{RESULTS \\ PARTICIPANTS}

Thirty-one DD patients were prescribed quetiapine; of these, two were lost-to-follow-up and three dropped out due to side-effects (Figure 1). Twenty-five SCZ patients were prescribed quetiapine; of these, two were lost-to-follow-up. Thirty-three SUD patients were prescribed quetiapine; of these, two were lost-to-follow-up, two dropped out due to side-effects, three dropped out due to relapse, and clinical data was missing for two patients. Therefore, LOCF analysis was available for 26, 23, and 24 patients in the DD, SCZ, and SUD group, respectively.

\section{SOCIODEMOGRAPHIC VARIABLES}

Significant differences were found for age $(F=5.5, p=0.006)$, gen$\operatorname{der}\left(\chi^{2}=7.1, p=0.03\right)$, and quetiapine dose $(F=22.1, p=0.0001)$ between the three groups (Table 1). By contrast, no significant differences were found between the groups in ethnicity, psychiatric diagnosis, type of substance(s) used, number of hospitalizations, and baseline antipsychotic. 


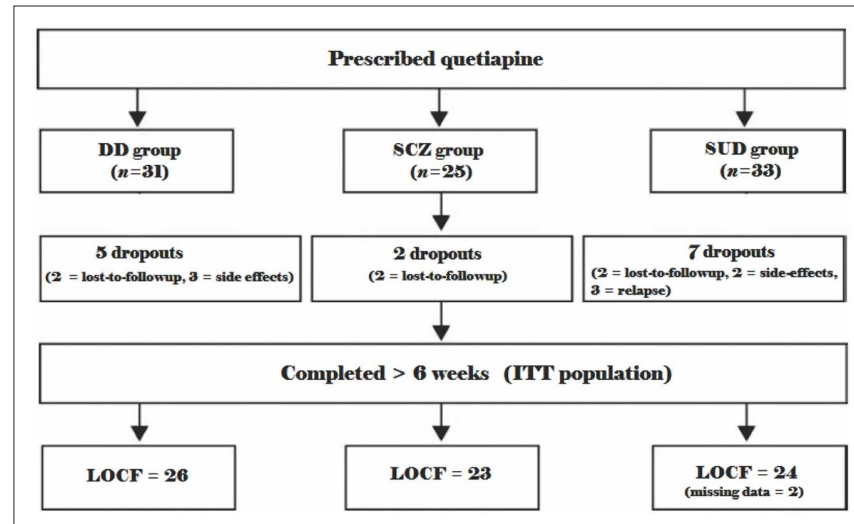

FIGURE 1 | Participant disposition. ITT, intention-to-treat; LOCF, lastobservation carried forward; DD, dual diagnosis group; SCZ, non-abusing schizophrenia group; SUD, non-schizophrenia substance abuser group.

\section{SUBSTANCE USE OUTCOMES}

Additionally, SUD patients had significantly higher SUD severity than DD patients at baseline $(F=11.3, p=0.002)$, but DD patients had significantly higher SUD severity at endpoint $(F=14.7, p<0.001$; Table 2). Moreover, SUD patients spent significantly more dollars per week on alcohol and drugs at baseline $(F=11.1, p=0.002)$, but there was no significant difference between the groups at endpoint. There was also a significant main effect of time for SUD severity $(F=106.4$, $p<0.001)$ and dollars per week $(F=21.5, p<0.001)$. Finally, SUD patients improved significantly more than DD patients in SUD severity $(F=41.7, p<0.001)$ and dollars per week $(F=16, p<0.001)$.

\section{NEUROLOGICAL SYMPTOMS}

Dual diagnosis patients had significantly more parkinsonism than SCZ patients at baseline $(F=3.6, p=0.03)$ and significantly more than SUD patients at endpoint $(F=4.2, p=0.02$; Table 3$)$. In addition, SUD patients had significantly higher akathisia scores than SCZ patients at baseline $(F=3.1, p=0.05)$, but not at endpoint. No significant differences were observed for dyskinesia at baseline or endpoint. Dystonia was not present in significant numbers in our sample (data not shown). Repeated measures analysis revealed that there was a main effect of time for parkinsonism $(F=9.5, p=0.003)$ and akathisia $(F=6.9$, $p=0.01$ ), but not dyskinesia. Changes in parkinsonism and dyskinesia did not differ significantly between the groups. Akathisia improved significantly more from baseline to endpoint in SUD relative to DD and SCZ patients $(F=5.3, p=0.02)$. The between-group differences in improvements in akathisia were no longer significant when changes in SUD outcomes were considered as covariates $(p=n . s)$. Sub-analyses of drug-specific effects revealed that improvements in akathisia in SUD patients were particular to cannabis abusers $(F=7.2, p=0.01)$. They also revealed that improvements in parkinsonism in $\mathrm{DD}$ patients were particular to stimulant abusers $(F=5.3, p=0.03)$.

\section{PSYCHIATRIC SYMPTOMS}

At baseline $(F=13.7, p<0.001)$ and endpoint, DD and SCZ patients had significantly higher PANSS negative scores compared to SUD patients ( $F=23.6, p<0.001$; Table 4$)$. In addition,
Table 1 | Sociodemographic variables.

\begin{tabular}{ll}
\hline Variable & $\begin{array}{l}\text { Statistics } \\
\text { (multiple comparisons)* }\end{array}$
\end{tabular}

\begin{tabular}{lll}
\hline \multicolumn{2}{l}{ AGE (YEARS) } & \\
DD & $30.5(9.5)$ & \\
SCZ & $40.6(12.4)$ & \\
SUD & $37.5(11)$ & \\
GENDER & & \\
DD & 24 Male, 2 female & $\chi^{2}=7.1, p=0.006(S C Z>D D)$ \\
SCZ & 15 Male, 8 female & \\
SUD & 15 Male, 9 female &
\end{tabular}

$\begin{array}{ll}\text { ETHNICITY } & \\ \text { DD } & \text { 24 Caucasian, } 2 \text { other } \\ \text { SCZ } & \text { 20 Caucasian, 3 other } \\ \text { SUD } & \text { 20 Caucasian, } 4 \text { other }\end{array}$

QUETIAPINE DOSE (MG/DAY)

$\begin{array}{lll}\text { DD } & 553.9(254.9) & F=22.1, p=0.0001 \\ & \text { (DD and SCZ > SUD) }\end{array}$

$\begin{array}{ll}\text { SCZ } & 478.3(272) \\ \text { SUD } & 150(117.7)\end{array}$

HOSPITALIZATIONS

$\begin{array}{lll}\text { DD } & 2.8(3) & F=0.8, p=0.4 \\ \text { SCZ } & 3.6(3.7) & \end{array}$

SUD -

\section{DIAGNOSIS}

$\begin{array}{ll}\text { DD } & \text { 15 SZ, 9 SA, 2 SF } \\ \text { SCZ } & \text { 13 SZ, 5 SA, 1 SF }\end{array}$

SUD S 5 SA, 1 SF

$\begin{array}{lll}\text { BASELINE ANTIPSYCHOTIC (ATYPICAL:TYPICAL:BOTH:DRUG-FREE) } \\ \text { DD } & 18: 4: 3: 1 & \chi^{2}=4, p=0.3 \\ \text { SCZ } & 14: 4: 0: 3 & \\ \text { SUD } & - & \end{array}$

\begin{tabular}{lll}
\multicolumn{3}{l}{ ALCOHOL ABUSE/DEPENDENCE } \\
DD & 12 Yes, 14 no & $\chi^{2}=0.3, p=0.6$ \\
SCZ & - & \\
SUD & 13 Yes, 11 no
\end{tabular}

CANNABIS ABUSE/DEPENDENCE

DD $\quad 15$ Yes, 11 no $\quad \chi^{2}=0.1, p=0.7$

SCZ

SUD 15 Yes, 9 no

\section{STIMULANT ABUSE/DEPENDENCE}

$\begin{array}{lll}\text { DD } & 9 \text { Yes, } 17 \text { no } & \chi^{2}=0.3, p=0.6 \\ \text { SCZ } & - & \\ \text { SUD } & 10 \text { Yes, 14 no }\end{array}$

\section{MULTI-SUBSTANCE ABUSE/DEPENDENCE}

$\begin{array}{lll}\text { DD } & 10 \text { Yes, } 16 \text { no } & \chi^{2}=2, p=0.2 \\ \text { SCZ } & - & \\ \text { SUD } & 14 \text { Yes } 10 \text { no } & \end{array}$

SZ, schizophrenia; SA, schizoaffective disorder; SF, schizophreniform disorder; DD, dual diagnosis group; SCZ, non-abusing schizophrenia group; SUD, non-schizophrenia substance abuser group.

*Bonferroni correction.

+ Missing data for one subject in SCZ group. 
Table 2 | Substance use disorder outcomes.

\begin{tabular}{|c|c|c|c|}
\hline Variable & Baseline & Endpoint & Statistics (multiple comparisons*) \\
\hline \multicolumn{4}{|c|}{ SUD SEVERITY } \\
\hline DD & $22.1(4.4)$ & $17.2(7.7)$ & \multirow{2}{*}{$\begin{array}{l}\text { Baseline: } F=11.3, p=0.002 \text {; time: } F=106.4, p<0.001 \text {; group } \times \text { time: } F=41.7 \text {, } \\
p<0.001 \text {; endpoint: } F=14.7, p<0.001\end{array}$} \\
\hline SUD & $28.3(7.9)$ & $7.1(10.4)$ & \\
\hline \multicolumn{4}{|c|}{ DOLLARS PER WEEK } \\
\hline DD & $93.4(65.4)$ & $61.8(60)$ & $\begin{array}{l}\text { Baseline: } F=11.1, p=0.002 \text {; time: } F=21.5, p<0.001 \text {; group } \times \text { time: } F=16 \text {, } \\
p<0.001 \text {; endpoint: } F=2.1, p=0.2\end{array}$ \\
\hline
\end{tabular}

DD, dual diagnosis group; SCZ, non-abusing schizophrenia group; SUD, non-schizophrenia substance abuser group.

*Bonferroni correction.

Table 3 | Neurological symptoms at baseline and endpoint.

\begin{tabular}{|c|c|c|c|}
\hline Variable & Baseline & Endpoint & Statistics (multiple comparisons*) \\
\hline \multicolumn{4}{|c|}{ PARKINSONISM } \\
\hline DD & $9.7(14.1)$ & $4.7(5.9)$ & Baseline: $F=3.6, p=0.03$ (DD > SCZ); time: $F=9.5, p=0.003$ \\
\hline SCZ & $2.5(2.6)$ & $2.1(2.4)$ & group $\times$ time: $F=1.8, p=0.2$; endpoint: $F=4.2, p=0.02$ (DD $>$ SUD) \\
\hline SUD & $5.7(6.9)$ & $1.5(3.4)$ & \\
\hline \multicolumn{4}{|c|}{ AKATHISIA } \\
\hline DD & $0.7(0.9)$ & $0.4(0.6)$ & Baseline: $F=3.1, p=0.05$ (SUD $>S C Z \#) ;$ time: $F=6.9$ \\
\hline SCZ & $0.6(1.1)$ & $0.7(1.2)$ & $p=0.01 ;$ group $\times$ time: $F=5.3, p=0.02($ SUD $>$ SCZ); endpoint: $F=1, p=0.4$ \\
\hline ISUD & $1.3(1.2)$ & $0.3(0.7)$ & \\
\hline \multicolumn{4}{|c|}{ DYSKINESIA } \\
\hline DD & $0.7(1.8)$ & $0.2(0.7)$ & Baseline: $F=2.7, p=0.07$; time: $F=1.1, p=0.3$; group $\times$ time: \\
\hline SCZ & $1.9(3.4)$ & $1.4(3.3)$ & $F=0.4, p=0.7 ;$ endpoint: $F=2.5, p=0.09$ \\
\hline SUD & $0.4(1.1)$ & $0.5(1.3)$ & \\
\hline
\end{tabular}

DD, dual diagnosis group; SCZ, non-abusing schizophrenia group; SUD, non-schizophrenia substance abuser group.

*Bonferroni correction.

${ }^{*}$ LSD correction.

Table 4 | Psychiatric symptoms at baseline and endpoint.

\begin{tabular}{|c|c|c|c|}
\hline Variable & Baseline & Endpoint & Statistics (multiple comparisons*) \\
\hline \multicolumn{4}{|c|}{ PANSS POSITIVE } \\
\hline DD & $18.3(4.3)$ & 15.6 (4) & Baseline: $F=1.4, p=0.3$; time: $F=38, p<0.001$; group $\times$ time: $F=5.3, p=0.007$ \\
\hline SCZ & $17.1(4.3)$ & $15.3(4.3)$ & (SUD > DD and SCZ); endpoint: $F=16.9, p<0.001$ (DD and SCZ > SUD) \\
\hline SUD & $16.2(5.4)$ & $10(2.7)$ & \\
\hline \multicolumn{4}{|c|}{ PANSS NEGATIVE } \\
\hline SUD & $12.5(4.8)$ & $8.9(3.2)$ & \\
\hline \multicolumn{4}{|c|}{ DEPRESSION } \\
\hline DD & $6.8(5)$ & $3.9(3.6)$ & Baseline: $F=3.2, p=0.05$ (DD > SCZ); time: $F=36.6, p<0.001$; group $\times$ time: \\
\hline SCZ & $3.6(4.6)$ & $1.2(1.6)$ & $F=1.1, p=0.4 ;$ endpoint: $F=5.7, p=0.005$ (DD > SCZ and SUD) \\
\hline SUD & $6(4.1)$ & $1.8(3.1)$ & \\
\hline
\end{tabular}

DD, dual diagnosis group; SCZ, non-abusing schizophrenia group; SUD, non-schizophrenia substance abuser group; PANSS, Positive and Negative Syndrome Scale. *Bonferroni correction.

depression scores were significantly higher in DD compared to SCZ patients at baseline $(F=3.2, p=0.05)$. Moreover, they were significantly higher in DD compared to SCZ and SUD patients at endpoint $(F=5.7, p=0.005)$. No differences were observed in
PANSS positive scores at baseline; however, PANSS positive symptoms were significantly higher in DD and SCZ patients at endpoint $(F=16.9, p<0.001)$. Repeated measures analysis revealed that was a significant main effect of time for PANSS positive $(F=38$, 
$p<0.001)$ and negative symptoms $(F=28.7, p<0.001)$ as well as depression $(F=36.6, p<0.001$; Table 4$)$. Changes in negative and depressive symptoms did not differ significantly between the groups (Table 4). However, PANSS positive symptoms improved significantly more in SUD patients from baseline to endpoint, compared to DD and SCZ patients $(F=5.3, p=0.007)$. There was no effect of age, gender, and dose when these variables were entered into the ANCOVA model. However, the finding of a greater improvement in positive symptoms in SUD patients disappeared after changes in SUD severity in time were considered as a covariate $(p=$ n.s).

\section{DISCUSSION}

The present study aimed to examine changes in substance use, as well as neurological symptoms and psychiatric symptoms in substance abusers with and without schizophrenia and in nonabusing schizophrenia patients undergoing 12-week treatment with quetiapine We found that SUD patients had a higher mean SUD severity, spent significantly more dollars weekly on alcohol and drugs at baseline and showed greater improvement in these variables, compared to DD patients. Nevertheless, at endpoint, there was no significant difference in dollars spent, but DD patients still had a higher mean SUD severity. Interestingly, DD patients had significantly higher parkinsonism and depression than SCZ patients at baseline and endpoint. On the other hand, we found that SUD patients had significantly more akathisia at baseline, improved more than SCZ patients and this was related to cannabis abuse/dependence. Finally, there were no significant differences in PANSS positive scores between the groups; however, SUD patients improved more and the differences were significant at endpoint.

In the present study, we found that SUD patients improved more in terms of SUD outcomes than DD patients. One explanation for this result could be that SUD patients had a significantly higher SUD severity at baseline, leading to the greater improvement. In addition, our SUD group began the study in detoxification, whereas our DD group were active users, suggesting that it was easier for the former patients to quit alcohol and/or drugs. Alternatively, these results suggest that it may be more difficult for schizophrenia patients to reduce or quit their substance use (Ziedonis et al., 2005). Importantly, DD patients still had a higher mean SUD severity than SUD patients at endpoint, despite spending similar amounts on alcohol and drugs. This finding is consistent with reports that substance abuse can have negative consequences on schizophrenia patients even when they use small amounts, infrequently (Ziedonis et al., 2005). It is also consistent with evidence of increased dopaminergic sensitivity in schizophrenia. Indeed, positron emission tomography (PET) studies have reported increased $\mathrm{D}_{2} / \mathrm{D}_{3}$ occupancy in schizophrenia patients in response to amphetamine challenge, relative to healthy controls (Laruelle et al., 1996; Abi-Dargham et al., 1998).

In terms of EPS, we found that DD patients had elevated parkinsonism at baseline, relative to SCZ and SUD patients, despite using significantly smaller quantities of alcohol and/or drugs. At endpoint, DD patients still had elevated parkinsonism relative to the other two groups, although they were taking similar amounts of these substances relative to SUD patients. Interestingly, a subanalysis revealed that improvements in parkinsonism were only significant in abusers of psychostimulants in the DD group. Obviously, the increase in parkinsonism in DD patients, relative to SUD patients, may be attributed to the fact that schizophrenia patients concomitantly take antipsychotics, which may interact with psychostimulants to increase parkinsonism (Potvin et al., 2006b; Maat et al., 2008). Indeed - when given acutely - cocaine and amphetamine stimulate striatal dopaminergic neurotransmission by blocking and reversing the dopamine transporter, respectively. However, their long-term abuse is associated with significant reduction in dopamine $\mathrm{D}_{2}$ receptor availability in the striatum that may last for months after detoxification, similar to the striatal dopaminergic deficit observed in Parkinson's disease (Volkow et al., 2004). Taken together, these results suggest that schizophrenia patients are more vulnerable to develop parkinsonism than SUD patients, even when taking small amounts of psychostimulants.

An unexpected result of the present study is the elevated akathisia at baseline in SUD patients. Intriguingly, a subanalysis revealed that the improvements in akathisia were found in cannabis abusers, which is consistent with reports of restlessness and physical tension/agitation among patients undergoing cannabis withdrawal (Kouri and Pope, 2000; Budney et al., 2003). Moreover, we found that akathisia improved significantly more in SUD patients, relative to SCZ patients, which is consistent with previous accounts of cannabinoid withdrawal. Overall, these results suggest that the endogenous cannabinoid system plays a role in the manifestation of akathisia, which may be related to its role in motor behavior (El Manira and Kyriakatos, 2010).

Analysis of psychiatric symptoms revealed that DD and SCZ patients had significantly more negative symptoms, relative to SUD patients at baseline and endpoint. This is consistent with evidence suggesting that negative symptoms are relatively unique to schizophrenia (Zhornitsky et al., 2010a). By contrast, we found that depressive symptoms were nearly twice as high in DD and over one and a half times higher in SUD compared to SCZ patients. This finding is in line with research showing that substance abuse is a risk factor for the development of depression (Lynskey et al., 2004; Falck et al., 2006; Pozzi et al., 2008) as well as with our meta-analysis of 3283 patients showing that addicted schizophrenia patients experience more severe depressive symptoms compared to non-abusing patients (Potvin et al., 2007). At study endpoint, depression scores were persistently elevated in DD patients. Taken together, these results are consistent with increased vulnerability in schizophrenia patients in response to drugs of abuse. Finally, at baseline - but not at endpoint - we found that all three groups had equally significant levels of positive symptoms, which is consistent with observations of elevated positive symptoms in non-schizophrenia substance abusers (Mauri et al., 2007; Lapworth et al., 2009). Interestingly, however, despite their high levels of positive symptoms, only two SUD patients responded to substance-induced psychosis criteria (SIPD; DSM-IV). Since the DSM-IV notes that a patient must have persistent delusions or hallucinations coupled with a lack of insight to be diagnosed with SIPD, we examined in more detail which PANSS positive items were most elevated at baseline in our SUD group. We found that the most elevated items (mean score $\approx 3$ ) were hostility, excitement and paranoia/suspiciousness; symptoms which may manifest during postintoxication or withdrawal but do not signify the presence of SIPD, 
according to the DSM-IV (Unnithan and Cutting, 1992; West and Gossop, 1994; Rosenthal et al., 1998; Mathias et al., 2008). Moreover, the fact that positive symptoms showed greater improvement in SUD patients, relative to DD and SCZ patients, is likely linked to their greater improvement in substance abuse outcomes. Taken together, our findings suggest that paranoia is not a symptom which reliably distinguishes between schizophrenia and SUD patients, when the latter individuals are undergoing withdrawal.

Improvements in neurologic and psychiatric symptoms did not differ between DD and SCZ patients, meaning that DD patients can improve in time as much as SCZ patients, as long as they significantly decrease their drug consumption - a finding that is consistent with previous reports (Conley et al., 1998; Swartz et al., 2008). Thus, it is not necessarily true that $\mathrm{DD}$ patients are doomed to have a worse prognosis than SCZ patients (Mueser et al., 1998; Negrete, 2003); rather, our results suggest that similar rates of improvements in psychiatric symptoms can be expected when DD patients diminish their substance use. However, in DD patients who maintain their substance use, this could prove otherwise. Indeed, there is evidence from non-pharmacological studies that psychotic patients who maintain their substance use have more severe depression, more positive symptoms, poorer functional outcome, and greater rates of relapse at 1 year follow-up, relative to non-users and those who maintain abstinence (Turkington et al., 2009).

The present study contains both strengths and limitations. Importantly, this is the first study of its kind to trace the evolution of substance abuse, neurological and psychiatric symptoms in DD, SCZ, and SUD patients undergoing a homogenous antipsychotic treatment. However, this pilot study was not powered to detect complex interactions between sociodemographic, psychiatric, neurologic, and

\section{REFERENCES}

Abi-Dargham,A., Gil, R., Krystal, J., Baldwin, R.M., Seibyl, J.P., Bowers, M., van Dyck, C. H., Charney, D. S., Innis, R. B., and Laruelle, M. (1998). Increased striatal dopamine transmission in schizophrenia: confirmation in a second cohort. Am. J. Psychiatry 155, 761-767.

Addington, D., Addington, J., and MatickaTyndale, E. (1993). Assessing depression in schizophrenia: the Calgary Depression Scale. Br. J. Psychiatry Suppl. 22, 39-44.

Addington, J., and Addington, D. (1997). Substance abuse and cognitive functioning in schizophrenia. J. Psychiatry Neurosci. 22, 99-104.

Arvanitis, L. A., and Miller, B. G. (1997). Multiple fixed doses of "Seroquel" (quetiapine) in patients with acute exacerbation of schizophrenia: a comparison with haloperidol and placebo. The Seroquel Trial 13 Study Group. Biol. Psychiatry 42, 233-246.

Aubin, G., Stip, E., Gélinas, I., Rainville, C., and Chapparo, C. (2009). Daily activities, cognition and community functioning in persons with schizophrenia. Schizophr. Res. 107, 313-318.

Bandelow, B., Chouinard, G., Bobes, J., Ahokas, A., Eggens, I., Liu, S., and
Eriksson, H. (2010). Extended-release quetiapine fumarate (quetiapine XR): a once-daily monotherapy effective in generalized anxiety disorder. Data from a randomized, double-blind, placebo- and active-controlled study. Int. J. Neuropsychopharmacol. 13, 305-320.

Barnes, T. R. (1989). A rating scale for drug-induced akathisia. Br. J. Psychiatry 154, 672-676.

Bersani, G., Gherardelli, S., Clemente, R., Di Giannantonio, M., Grilli, A., Conti, C. M., Exton, M. S., Conti, P., Doyle, R., and Pancheri, P. (2005). Neurologic soft signs in schizophrenic patients treated with conventional and atypical antipsychotics. J. Clin. Psychopharmacol. 25, 372-375.

Budney, A. J., Moore, B. A., Vandrey, R. G., and Hughes, J. R. (2003). The time course and significance of cannabis withdrawal. J. Abnorm. Psychol. 112, 393-402.

Chouinard, G., Ross-Chouinard, A., Annable, L., and Jones, B. (1980). The extrapyramidal symptom rating scale. Can. J. Neurol. Sci. 7, 233.

Conley, R. R., Kelly, D. L., and Gale, E. A. (1998). Olanzapine response in treatment-refractory schizophrenic

SUD variables. The study is also limited because the design does not permit us to deduce whether or not quetiapine played a significant role in the improvements in psychiatric symptoms in SUD patients. Interestingly, there is evidence to suggest that low-dose quetiapine is a highly effective anxiolytic and antidepressant and may possess mild antipsychotic activity as well (Fabre et al., 1995; Arvanitis and Miller, 1997; Cutler et al., 2009; Bandelow et al., 2010; see Zhornitsky et al., 2011 for review). However, we entered dose into the ANCOVA model and it showed no significant effect for any of our results. Other potential confounds such as age and gender also did not affect our results. By contrast, SUD severity was a significant factor in the ANCOVA models and is likely related to the fact that our SUD patients were recruited when they entered into detoxification, whereas our DD patients were active users. Thus, differences in changes in neurological and psychiatric symptoms between SUD patients and the other two groups seem to be intoxication/withdrawal-related phenomena. Future studies should take more frequent measurements (e.g., every 3 weeks) of psychiatric symptoms and EPS, in order to better elucidate the temporal relationship in improvements of these variables. The contribution of quetiapine in the psychiatric and neurologic symptoms reported here will also need to be elucidated.

\section{ACKNOWLEDGMENTS}

This trial was financed via an academic partnership between the Louis-H Lafontaine Foundation AstraZeneca Pharmaceuticals. Emmanuel Stip is holder of the Eli Lilly Canada Chair of Schizophrenia from the University of Montreal. Stéphane Potvin is holder of a Junior 1 researcher scholarship from the Fonds de Recherche en Santé du Québec.

patients with a history of substance abuse. Schizophr. Res. 33, 95-101.

Cutler, A. J., Montgomery, S. A., Feifel, D., Lazarus, A., Aström, M., and Brecher, M. (2009). Extended release quetiapine fumarate monotherapy in major depressive disorder: a placebo- and duloxetine-controlled study. J. Clin. Psychiatry 70, 526-539.

Eaton, W. W., Martins, S. S., Nestadt, G., Bienvenu, O. J., Clarke,D., and Alexandre, P. (2008). The burden of mental disorders. Epidemiol. Rev. 30, 1-14.

El Manira, A., and Kyriakatos, A. (2010). The role of endocannabinoid signaling in motor control. Physiology (Bethesda) 25, 230-238.

Fabre, L. F. Jr., Arvanitis, L., Pultz, J., Jones, V. M., Malick, J. B., and Slotnick, V. B. (1995). ICI 204,636, a novel, atypical antipsychotic: early indication of safety and efficacy in patients with chronic and subchronic schizophrenia. Clin. Ther. 17, 366-378.

Falck, R. S., Wang, J., Carlson, R. G., and Siegal, H. A. (2006). Prevalence and correlates of current depressive symptomatology among a community sample of MDMA users in Ohio. Addict. Behav. 31, 90-101.

Green, A. I., Burgess, E. S., Dawson, R., Zimmet, S. V., and Strous, R. D.
(2003). Alcohol and cannabis use in schizophrenia: effects of clozapine vs. risperidone. Schizophr. Res. 60, 81-85.

Harrison, I., Joyce, E. M., Mutsatsa, S. H., Hutton, S. B., Huddy, V., Kapasi, M., and Barnes, T. R. (2008). Naturalistic follow-up of co-morbid substance use in schizophrenia: the West London first-episode study. Psychol. Med. 38, 79-88.

Kampman, K. M., Pettinati, H. M., Lynch, K. G., Whittingham, T., Macfadden, W., Dackis, C., Tirado, C., Oslin, D. W., Sparkman, T., and O'Brien, C. P. (2007). A double-blind, placebocontrolled pilot trial of quetiapine for the treatment of Type A and Type B alcoholism. J. Clin. Psychopharmacol. 27, 344-351.

Kay, S. R., Fiszbein, A., and Opler, L. A. (1987). The Positive and Negative Syndrome Scale (PANSS) for schizophrenia. Schizophr. Bull. 13, 261-276.

Koob, G. F., and Le Moal, M. (2001). Drug addiction, dysregulation of reward, and allostasis. Neuropsychopharmacology 24, 97-129.

Kouri, E. M., and Pope, H. G. Jr. (2000). Abstinence symptoms during withdrawal from chronic marijuana use. Exp. Clin. Psychopharmacol.8,483-492. 
Lahmek, P., Berlin, I., Michel, L., Berghout, C., Meunier, N., and Aubin, H.J. (2009). Determinants of improvement in quality of life of alcohol-dependent patients during an inpatient withdrawal programme. Int. J. Med. Sci. 6, 160-167.

Lapworth, K., Dawe, S., Davis, P., Kavanagh, D., Young, R., and Saunders, J. (2009). Impulsivity and positive psychotic symptoms influence hostility in methamphetamine users. Addict. Behav. 34, 380-385.

Laruelle, M., Abi-Dargham, A., van Dyck, C. H., Gil, R., D'Souza, C. D., Erdos, J., McCance, E., Rosenblatt, W., Fingado, C.,Zoghbi, S. S., Baldwin, R. M., Seibyl, J. P., Krystal, J. H., Charney, D. S., and Innis, R. B. (1996). Single photon emission computerized tomography imaging of amphetamine-induced dopamine release in drug-free schizophrenic subjects. Proc. Natl. Acad. Sci. U.S.A. 93, 9235-9240.

Lee, K. U., Jeon, Y. W., Lee, H. K., and Jun, T. Y. (2009). Efficacy and safety of quetiapine for depressive symptoms in patients with schizophrenia. Hum. Psychopharmacol. 24, 447-452.

Lieberman, J. A., Stroup, T. S., McEvoy, J. P., Swartz, M. S., Rosenheck, R. A., Perkins, D. O., Keefe, R. S., Davis, S. M., Davis, C. E.,Lebowitz, B.D.,Severe, J., and Hsiao, J. K. (2005). Effectiveness of antipsychotic drugs in patients with chronic schizophrenia. N. Engl.J.Med.353, 1209-1223.

Littrell, K. H., Petty, R. G., Hilligoss, N. M., Peabody,C.D., and Johnson, C.G. (2001). Olanzapine treatment for patients with schizophrenia and substance abuse. J. Subst. Abuse Treat. 21, 217-221.

Lynskey, M. T., Glowinski, A. L., Todorov, A. A., Bucholz, K. K., Madden, P. A., and Nelson, E. C., Statham, D. J., Martin, N. G., Heath, A. C. (2004). Major depressive disorder, suicidal ideation, and suicide attempt in twins discordant for cannabis dependence and early-onset cannabis use. Arch. Gen. Psychiatry 61, 1026-1032.

Maat, A., Fouwels, A., and de Haan, L. (2008). Cocaine is a major risk factor for antipsychotic induced akathisia, parkinsonism and dyskinesia. Psychopharmacol. Bull. 41, 5-10.

Martinotti, G., Andreoli, S., Di Nicola, M., Di Giannantonio, M., Sarchiapone, M., and Janiri, L. (2008). Quetiapine decreases alcohol consumption, craving, and psychiatric symptoms in dually diagnosed alcoholics. Hum. Psychopharmacol. 23, 417-424.

Martinotti, G., Di Nicola, M., Di Giannantonio, M., and Janiri, L. (2009). Aripiprazole in the treatment of patients with alcohol dependence: a doubleblind, comparison trial vs. naltrexone. J. Psychopharmacol. 23, 123-129.

Martinotti, G., Di Nicola, M., and Janiri, L. (2007). Efficacy and safety of aripiprazole in alcohol dependence. Am. J. Drug Alcohol Abuse 33, 393-401.

Mathias, S., Lubman, D. I., and Hides, L. (2008). Substance-induced psychosis: a diagnostic conundrum. J. Clin. Psychiatry 69, 358-367.

Mauri, M. C., Volonteri, L. S., Fiorentini, A., Pirola, R., and Bareggi, S. R. (2007). Two weeks' quetiapine treatment for schizophrenia, drug-induced psychosis and borderline personality disorder: a naturalistic study with drug plasma levels. Expert Opin. Pharmacother. 8, 2207-2213.

Mueser, K. T., Drake, R. E., and Wallach, M.A. (1998). Dual diagnosis: a review of etiological theories. Addict. Behav. $23,717-734$.

Nakamura, M., Ogasa, M., Guarino, J., Phillips, D., Severs, J., Cucchiaro, J., and Loebel, A. (2009). Lurasidone in the treatment of acute schizophrenia: a double-blind, placebo-controlled trial. J. Clin. Psychiatry 70, 829-836.

Negrete, J. C. (2003). Clinical aspects of substance abuse in persons with schizophrenia. Can. J. Psychiatry 48, 14-21.

Patterson, T. L., Klapow, J. C., Eastham, J. H., Heaton, R. K., Evans, J.D., Koch, W. L., and Jeste, D. V. (1998). Correlates of functional status in older patients with schizophrenia. Psychiatry Res. 80, 41-52.

Potvin, S., Blanchet, P., and Stip, E. (2009). Substance abuse is associated with increased extrapyramidal symptoms in schizophrenia: a meta-analysis. Schizophr. Res. 113, 181-188.

Potvin,S.,Sepehry,A.A., and Stip, E. (2007). Meta-analysis of depressive symptoms in dual-diagnosis schizophrenia. Aust. N. Z. J. Psychiatry 41, 792-799.

Potvin, S., Stip, E., Lipp, O., Elie, R., ManciniMarië, A., Demers, M. F., Roy, M. A., Bouchard, R. H., and Gendron, A. (2006a). Quetiapine in patients with comorbid schizophrenia-spectrum and substance use disorders: an open-label trial.Curr.Med.Res. Opin. 22, 1277-1285.

Potvin, S., Pampoulova, T., ManciniMarie, A., Lipp, O., Bouchard, R. H., and Stip, E. (2006b). Increased extrapyramidal symptoms in patients with schizophrenia and a comorbid substance use disorder. J. Neurol. Neurosurg. Psychiatry 77, 796-798.

Pozzi, G., Martinotti, G., Reina, D., Dario, T., Frustaci, A., Janiri, L., and Bria, P. (2008). The assessment of post-detoxification anhedonia: influence of clinical and psychosocial variables. Subst. Use Misuse 43, 722-732.

Regier, D. A., Farmer, M. E., Rae, D. S., Locke, B. Z., Keith, S. J., Judd, L. L., and Goodwin, F. K. (1990). Comorbidity of mental disorders with alcohol and other drug abuse. Results from the Epidemiologic Catchment Area (ECA) Study. JAMA 264, 2511-2518.
Rizkallah, E., Stip, E., Zhornitsky, S., Pampoulova, T., Gendron, A., Rompre, P. P., Chiasson, J. P., and Potvin, S. (2010). Clinical evolution of patients with substance use disorders during treatment with quetiapine: a 12-week, open-label, naturalistic trial. Expert Opin. Pharmacother. 11, 2947-2951.

Rosenthal, R. N., Perkel, C., Singh, P., Anand, O., and Miner, C. R. (1998). A pilot open randomized trial of valproate and phenobarbital in the treatment of acute alcohol withdrawal. Am J. Addict. 7, 189-197.

Sobell, L. C., and Sobell, M. B. (1992) "Timeline follow-back: a technique for assessing self-reported ethanol consumption," in Measuring Alcoho Consumption: Psychosocial and Biological Methods, eds J. Allen and R. Z. Litten (Totowa, NJ: Humana Press), 41-72.

Swartz, M. S., Wagner,H.R.,Swanson, J.W., Stroup, T. S., McEvoy, J. P., Reimherr F., Miller, D. D., McGee, M., Khan, A. Canive, J. M., Davis, S. M., Hsiao, J. K. and Lieberman, J.A. (2008). The effectiveness of antipsychotic medications in patients who use or avoid illicit substances: results from the CATIE study. Schizophr. Res. 100, 39-52.

Turkington, A., Mulholland, C. C., Rushe, T. M., Anderson, R., McCaul, R., Barrett, S. L., Barr, R. S., and Cooper, S. J. (2009). Impact of persistent substance misuse on 1-year outcome in first-episode psychosis. Br. J.Psychiatry $195,242-248$

Unnithan, S. B., and Cutting, J. C. (1992). The cocaine experience: refuting the concept of a model psychosis? Psychopathology 25, 71-78.

van Nimwegen, L. J., de Haan, L., van Beveren, N. J., van der Helm, M. van den Brink, W., and Linszen, D. (2008). Effect of olanzapine and risperidone on subjective well-being and craving for cannabis in patients with schizophrenia or related disorders: a double-blind randomized controlled trial. Can. J. Psychiatry 53, 400-405.

Villalta-Gil, V., Vilaplana, M., Ochoa, S., Haro, J. M., Dolz, M., Usall, J. Cervilla, J., and NEDENA Group. (2006). Neurocognitive performance and negative symptoms: are they equal in explaining disability in schizophrenia outpatients? Schizophr. Res. 87, 246-253.

Volkow, N. D., Fowler, J.S., Wang, G. J., and Swanson, J. M. (2004). Dopamine in drug abuse and addiction: results from imaging studies and treatment implications. Mol. Psychiatry 9, 557-569.

Weiden, P. J. (2007). EPS profiles: the atypical antipsychotics are not all the same. J. Psychiatr. Pract. 13, 13-24.

West, R., and Gossop, M. (1994). Overview: a comparison of withdrawal symptoms from different drug classes. Addiction 89, 1483-1489.

Zhornitsky, S., Potvin, S., Moteshafi, H., Dubreucq, S., Rompre, P. P., and Stip, E. (2011). Dose-response and comparative efficacy and tolerability of quetiapine across psychiatric disorders: a systematic review of the placebo-controlled monotherapy and add-on trials. Int. Clin. Psychopharmacol. [Epub ahead of print].

Zhornitsky, S., Stip, E., Pampoulova, T., Rizkallah, E., Lipp, O., Bentaleb, L. A., Chiasson, J. P., and Potvin, S. (2010a). Extrapyramidal symptoms in substance abusers with and without schizophrenia and in nonabusing patients with schizophrenia. Mov. Disord. 25, 2188-2194.

Zhornitsky, S., Rizkallah, E., Pampoulova, T., Chiasson, J. P., Stip, E., Rompré,P.P., and Potvin, S. (2010b). Antipsychotic agents for the treatment of substance use disorders in patients with and without comorbid psychosis. J. Clin. Psychopharmacol. 30, 417-424.

Ziedonis, D. M., Smelson, D., Rosenthal, R. N., Batki, S. L., Green, A. I., Henry, R. J., Montoya, I., Parks, J., and Weiss, R. D. (2005). Improving the care of individuals with schizophrenia and substance use disorders: consensus recommendations. J. Psychiatr. Pract. 11,315-339.

Conflict of Interest Statement: The authors declare that the research was conducted in the absence of any commercial or financial relationships that could be construed as a potential conflict of interest.

Received: 29 January 2011; accepted: 18 April 2011; published online: 13 May 2011.

Citation: Zhornitsky S, Stip E, Desfossés J, Pampoulova T, Rizkallah É, Rompré P-P, Bentaleb LA, Lipp O, Chiasson J-P, Gendron A and Potvin S (2011) Evolution of substance use, neurological and psychiatric symptoms in schizophrenia and substance use disorder patients: a 12-week, pilot, case-control trial with quetiapine. Front. Psychiatry 2:22. doi: 10.3389/ fpsyt.2011.00022

This article was submitted to Frontiers in Addictive Disorders, a specialty of Frontiers in Psychiatry.

Copyright (ㅇ 2011 Zhornitsky, Stip, Desfossés, Pampoulova, Rizkallah, Rompré Bentaleb, Lipp, Chiasson, Gendron and Potvin. This is an open-access article subject to a non-exclusive license between the authors and Frontiers Media SA, which permits use, distribution and reproduction in other forums, provided the original authors and source are credited and other Frontiers conditions are complied with. 